\title{
Cintilografia Miocárdica para Pesquisa de Infarto Agudo do Miocárdio (IAM)
}

Em algumas situações especiais, o diagnóstico do IAM pode ser dificultado pela presença de alterações prévias no ECG (BRE, por exemplo) e/ou ocorrência recente de manipulação externa ou interna do coração (ressuscitação cardiorrespiratória / cirurgia cardíaca). Nessas ocasiões, a imagem com pirofosfato-99mTc pode ser útil. Como durante o processo evolutivo do IAM ocorre grande influxo de cálcio para o espaço intracelular, a concentração do 99mTc-pirofosfato no miocárdio acompanha esta elevação intracelular, apresentando um pico máximo de captação cerca de 48-72 horas após o evento agudo.

Além do uso no diagnóstico da necrose miocárdica que ocorre no IAM, o pirofosfato-99mTc é ocasionalmente utilizado na detecção da amiloidose cardíaca e alguns trabalhos mostram a sua captação em músculo inflamado, como ocorre na miocardite.

A antimiosina monoclonal murínica, marcada com índio-111 (antimiosina- ${ }^{111} \mathrm{In}$ ), liga-se seletivamente aos miócitos irreversivelmente lesados, podendo ser utilizada para a detecção da necrose, mas apresentando limitações para sua utilização, não só relacionadas à pouca disponibilidade, mas também à necessidade de aguardar-se de 24 a 48 horas para a obtenção de imagens com qualidade adequada.

O glucarato- ${ }^{99 m}$ Tc é um análogo da glicose, que se acumula rapidamente na área do infarto recente, podendose obter suas imagens 30 minutos após a injeção. A positividade do exame é tempo-dependente, não estando presente após 9 horas do início da dor precordial. Este agente ainda não está disponível comercialmente.

\section{REFERÊNCIAS}

1. De Puey Eg, Port S, Wackers FJT et al. Nonperfusion applications in nuclear cardiology: report of a task force of the American Society of Nuclear Cardiology. J Nucl Cardiol 1998; 5: 218-31.
2. Gertz MA, Brown ML, Hauser MF, Kyle RA. Utility of technetium Tc 99m pyrophosphate bone scanning in cardiac amyloidosis. Arch Intern Med 1987; 147:1039-44. 